\title{
Transatlantica
}

Revue d'études américaines. American Studies Journal

\section{Yves Carlet. Stephen Crane. Les couleurs de l'angoisse.}

Paris : Belin, 2002. 128 p.

\section{Claude Dorey}

\section{(2) OpenEdition}

Journals

Édition électronique

URL : http://journals.openedition.org/transatlantica/724

DOI : 10.4000/transatlantica.724

ISSN : $1765-2766$

Éditeur

AFEA

Référence électronique

Claude Dorey, "Yves Carlet. Stephen Crane. Les couleurs de l'angoisse. », Transatlantica [En ligne], 1 | 2003, mis en ligne le 05 avril 2006, consulté le 29 avril 2021. URL : http://journals.openedition.org/ transatlantica/724; DOI : https://doi.org/10.4000/transatlantica.724

Ce document a été généré automatiquement le 29 avril 2021

\section{(c) (i)}

Transatlantica - Revue d'études américaines est mis à disposition selon les termes de la licence Creative Commons Attribution - Pas d'Utilisation Commerciale - Pas de Modification 4.0 International. 


\section{Yves Carlet. Stephen Crane. Les couleurs de l'angoisse.}

Paris : Belin, 2002. 128 p.

Claude Dorey

1 Il est heureux que les cendres de Stephen Crane entrent au panthéon de Marc Chénetier. Yves Carlet, l'ordonnateur, nous convie au «survol» d'une œuvre qu'il décrit comme un «commencement avorté». Le trajet s'effectue au moyen d'oppositions binaires. Un axe de polarités contraires est, d'entrée, posé : d'un côté, la ligne morale de l'honnêteté réaliste, de l'autre, celle, baroque, du conteur ironique et nostalgique. Néanmoins une «cohérence profonde» dessinée par des «motifs obsédants» serait repérable. L'absence de solution de continuité entre l'œuvre journalistique et les romans favoriserait la mise en évidence de celle-ci. Crane ferait son apprentissage en composant des croquis journalistiques, le plus souvent consacrés à "l'autre moitié ", où déjà bouillonneraient les « images obsédantes des romans et des nouvelles ». Le romancier "précéderait donc le journaliste ». Crane se différencierait cependant des naturalistes purs et durs tels que Zola, car il décrit des plongées dans les abîmes de la désocialisation quand le Français propose une représentation précise d'individus appartenant à des groupes sociaux affrontés. Enfin les enquêtes de Crane sont travaillées par une tension profonde entre pathos et ironie. Evoquant Maggie, Carlet poursuit sa réflexion sur la question du naturalisme. Si la force de l'environnement n'est pas absente, le déterminisme de celui-ci s'exerce sous une forme plus cauchemardesque et ténébreuse qu'économique et sociale. Clôture et exclusion, enfermement et violence en constituent les lignes de force. La conscience de classe est peu présente, même si «c'est la censure sociale et religieuse qui est dans ce roman la racine du mal», ou encore «le conformisme bourgeois ». Face à celui-ci, la «volonté parodique prend le pas sur la logique naturaliste». Ce texte constituerait une « révolution copernicienne » du projet naturaliste, la fiction s'y donnant moins comme enquête sociale que comme "carnet intime " où s'inscrivent les affects, les illusions d'une «fille des rues». Toujours à propos de Maggie, l'étiquette impressionniste est contestée. A partir des trois scènes situées au music-hall, Carlet montre comment le passage de l'euphorie au cauchemar est manifesté par les altérations de la perception, 
dans un vertige expressionniste aiguisé par une syntaxe syncopée et instable. George's Mother est ensuite évoqué, la symétrie formelle et thématique des deux textes imposant un parallèle qui permet, selon Carlet, de situer les limites du génie de Crane. Le deuxième chapitre s'ouvre sur les Sullivan County Sketches, plongées du « little man » fanfaron et de ses acolytes au cœur d'une Amérique primitive et déjà désuète. Ces textes constitueraient un "laboratoire où s'élaborent diverses stratégies narratives »: la parodie empruntée aux humoristes de l'Ouest, la parodie appliquée aux topos à la Cooper, la persistance obsédante du «fantasme phobique " que l'ironie vient ensuite vider de sa substance. Ces textes porteraient en germe bien des motifs de Red Badge, notamment l'échange de regards entre vif et mort, et l'échange de statut entre animé et inanimé. Viennent ensuite les textes de l'Ouest et du Mexique, notamment Nebraska's Bitter Fight for Life, reportage sur lâcheté et héroïsme devant les plaies infligées à l'homme par le feu de la sécheresse puis la vitrification d'un blizzard. Ce texte appelle dans son sillage thématique The Blue Hotel, où l'on voit un blizzard agréger quatre voyageurs dans un drôle de lieu. Le caractère énigmatique de ce récit de fiction excite les pulsions interprétatives. Celles qu'évoque Carlet sont peu convaincantes, notamment celle de Benfey. D'autres récits de l'Ouest et des textes sur le Mexique sont proposés à l'observation, soit dans la perspective des motifs obsédants, particulièrement la peur, soit dans celle de la déconstruction parodique. Entrent aussi dans cette séquence les textes de l'Ouest écrits trois ans plus tard, notamment The Bride Comes to Yellow Sky, brièvement traité. Un Ouest de la «frontière moribonde » et de la méprise y est mis en scène où Crane jouerait avec les codes narratifs des récits populaires sans toutefois renoncer à un fond nostalgique, deux voix se croiseraient, «l'une stridente, l'autre hésitante ».

Le troisième chapitre se consacre principalement à Red Badge. Plus qu'un roman de guerre, ce serait une "fable sur la peur " où s'engouffrent les motifs obsédants. A nouveau est posée la question du naturalisme, du degré de déterminisme. Pour mesurer celui-ci, une comparaison avec Norris est proposée. Avec Red Badge l'antagonisme thématique et structurel entre impuissance individuelle et toute-puissance de la machine de guerre reconduit le commentateur à la question des motifs obsédants, notamment ceux de l'intégration-enfermement et de la dissociation-amputation, de la réification des humains et de l'animation de l'environnement. Une caractérisation naturaliste serait reconnaissable au fait qu'Henry cherche son salut au sein de la nature et que celle-ci ne lui offre guère que des entraves. Outre cela, en campant un cadavre détaillé au cœur du repli forestier, Crane retrouverait le regard clinique d'un Zola, à cette différence que l'Américain n'aurait pas complètement abjuré les tentations du romantisme puisqu'il fait de cette rencontre une scène de fascination. Crane serait alors un « naturaliste et un décadent » qui utilise les « schèmes naturalistes » et les met au service de l'ironie. La question de Red Badge, roman d'initiation, est ensuite posée. Sont évoquées les interprétations qui font de RB une allégorie chrétienne ou un triptyque mythique ou encore une série de rites de passage ramenant la brebis égarée au bercail, sans oublier la lecture Jungienne de Cazemajou. Enfin la logique paradoxale qui anime tout le parcours du héros reconduit le commentateur à la question de l'ironie. Une pédagogie négative jaillirait de celle-ci, le fanfaron exalté prenant la mesure de son erreur glorieuse et de sa propre insignifiance. Où l'on retrouverait le nada de Hemingway. Surgit ensuite l'incontournable querelle relative à l'impressionnisme de Crane, vite close, Dieu merci, au profit de considérations sur la 
modernité de cette écriture. Il est alors question de «techniques protofilmiques »- pratique singulière du montage, représentation du mouvement etc ; autre nouveauté, cet art se vouerait à la représentation du «paradoxe de la perception en temps de crise», "expérience disjonctive» caractérisée par le retard. L'expressionnisme est à nouveau convoqué et Kandinsky aperçu à l'horizon. Ce chapitre se clôt par l'évocation d'autres récits de guerre comparés à des "eaux-fortes".

La conclusion générale se contente d'effleurer les poèmes, au motif que ceux-ci obéiraient à une implacable double contrainte: une affirmation quasi sacrilège d'autonomie face à un Dieu hégémonique et une inclination vers la parabole biblique à la Bunyan. Carlet oppose alors la « fadeur, la vacuité de l'univers poétique de Crane » à «l'intensité de son univers fictionnel », propos qu'il illustre via The Open Boat, texte de l'expérience, mais "décanté » par la ressouvenance et thématiquement proche des poèmes. C'est, de loin, la meilleure partie de cette étude. A partir de plusieurs citations s'élabore une analyse de cet "art poétique » de la fiction où se mêlent l'exigence de fidélité au réalisme brut et le souci de traduire la subjectivité des impressions. «La ligne de fuite qui permet la fusion entre ces deux démarches ... est celle du regard». On retrouverait dans ce texte la "révolte métaphysique des poèmes" mais ici la dérision enlèverait au sacré son caractère pesant. Carlet pointe les glissements entre le collectif et l'individuel dans ce récit d'une expérience solitaire et partagée où l'ironie fouette l'apitoiement sur soi. Pour conclure, cette étude fait souvent mouche. On ne saurait qu'approuver sa réticence vis à vis des clichés diagnostiques que charrie l'œuvre de Crane. Un regard pénétrant est porté sur les forces motrices et inhibitrices que constituent les motifs obsédants en liaison avec l'ironie. L'orientation générale nous paraît juste. On pourra ne pas toujours approuver le corpus privilégié et les relégations qui en découlent, mais le format est si bref. Une réserve majeure, cependant, le propos aurait gagné à prendre en compte des travaux plus récents, l'un d'eux notamment, effectué sous la direction de Michel Gresset. Ce travail propose une interprétation originale fondée sur une analyse approfondie des motifs obsédants. Elle met en évidence la présence d'une fantasmagorie insistante qui donne à l'œuvre sa dynamique mais la contraint dans des schèmes restreints. Selon cette lecture, la scène princeps, scène de fascination, est la rencontre du fils avec le cadavre tyrannique du père et toute l'œuvre se joue et se déjoue à partir de ce face à face. « $\mathrm{P}(\mathrm{l}) \mathrm{op}$ !» (The Upturned Face).

\section{INDEX}

Thèmes : Recensions 
AUTEUR

CLAUDE DOREY

Université Paris X-Nanterre 